BANGLADESH J CHILD HEALTH 2009; VOL 33 (3): 90-99

\title{
Non-convulsive Status Epilepticus in Children, Electro-Clinical Profile and Response To A Specific Treatment Protocol
}

\author{
SELINA HUSNA BANU ${ }^{1}$, MOSTAFA MAHBUB $^{2}$, AZM MOSHIUL AZAM $^{3}$, SHIPRA RANI $^{4}$, NAILA Z KHAN $^{5}$
}

\begin{abstract}
Objective: Non-convulsive status epilepticus (NCSE) is an under-diagnosed neurological condition. We report the electroclinical profile and treatment outcome of children diagnosed with NCSE.

Methods: Total 45 children were identified with NCSE at the EEG laboratory from September 2004 to January 2009. Their presenting complaints, past clinical and birth related information were meticulously recorded. On diagnosis the referring physicians were requested to repeat the test after starting treatment. Later they were treated with a specific protocol on admission at the Neurology Unit of Dhaka Shishu Hospital. Two lines of treatment with daily routine EEGs were introduced. Electroclinical outcome were recorded on discharge. The children are still on regular follow up to record the long-time result of the protracted treatment.
\end{abstract}

Results: Motor regression and postural problem were the primary complaints in the majority $(37 / 45,82 \%)$ followed by involuntary movement, muscle twitching, jerks or frequent fall $(31 / 45,69 \%)$, speech regression (29, 64\%) and change of usual behavior (25/45, 56\%). Prior major seizures (generalized tonic clonic, tonic or clonic) was reported in 32/45, 71\%, within 1 week to 1 year. Initial diagnosis was multiple including hysteric conversion reaction, post-ictal regression or neuro-degenerative disorder. None was suspected with NCSE. Diagnosis was confirmed by the EEG finding of continuous generalized (82\%) or localized (18\%) slow spike-wave complexes. On protracted treatment, $82 \%$ achieved the target.

Conclusion: NCSE is common in children, may occur de-novo. The variable phenotype may lead to erroneous diagnosis. A protracted treatment protocol is suggested. Further reporting on this issue will help to assist the clinicians for early-diagnosis and treatment.

Key words: non-convulsive status epilepticus, electro-clinical diagnosis, motor functional regression, speech regression, behavioural changes.

\section{Introduction}

Non-convulsive status epilepticus (NCSE) is defined as change of mental and functional status from the baseline for at least 30-60 minutes associated with

1. Clinical Neurophysiologist and Epileptologist, Assistant Professor, Neurosciences Unit, ICH and SSF Hospital, Mirpur, Dhaka

2. Assistant Professor, Child Development and Neurology Unit, Bangladesh Institute of Child Health, Dhaka Shishu Hospital, Dhaka

3. Junior Consultant, Child Development and Neurology Unit, Dhaka Shishu Hospital, Dhaka

4. Senior Technologist, EEG Laboratory, Comfort Diagnostic Centre, 169, Green Road, Dhaka

5. Professor and Head, Child Development and Neurology Unit, Bangladesh Institute of Child Health, Dhaka Shishu Hospital, Dhaka

Correspondence: Dr. Selina Husna Banu continuous or near-continuous slow spike wave discharges on electroencephalogram (EEG), and in the absence of clonic, tonic or tonic clonic seizures ${ }^{1}$.

It may occur following generalized convulsion, in comatose patient, as manifestation of a variety of epilepsy syndrome or may present de-novo. The change of behaviour and motor functional state is often misdiagnosed particularly in children and delay suspicion of such brain dysfunction.

Like the diagnostic difficulties, the treatment for NCSE is also non-conclusive. There are reports where conventional treatment for convulsive status epilepticus (CSE), i.e., start with i.v. benzodiazepines, (diazepam/ lorazepam), if not controlled i.v. phenytoine, phenobarbital and then general anasthesia 
are used ${ }^{2,3}$ but not conclusive. Some reported precipitation or worst effect on treatment with benzodiazepines or diazepam ${ }^{4,5}$.

There is lack of information about the diagnostic criteria and treatment in children particularly from the developing countries. Objective of this study was to compile the clinical profile and EEG features of the children diagnosed with NCSE and to report the shorttime outcome of a specific treatment protocol.

\section{Materials and Methods \\ Participants}

Patients were selected from the paediatric EEG laboratory of a privet clinic in Dhaka during the period of September 2004 to January 2009. NCSE was diagnosed based on the specific EEG criteria of nearcontinuous slow epileptiform discharges and the clinical condition of the child, i.e., regression of baseline motor function, behaviour for a prolonged duration and history of seizures. Children with EEG feature of continuous or near continuous slow spike wave complex discharges (1-2.5 cycle per second, of amplitude100 Uv and above) in the routine EEG for 30 minutes without any history of overt convulsion during the previous 48 hours were included.

Those with continuous but periodic lateralized epileptiform discharges (PLED) in the EEG were excluded as they are known to have specific aetiology, i.e., white or gray matter lesions, associated with coma and known poor outcome $\mathrm{e}^{6-8}$.

\section{EEG recording}

As per laboratory protocol, routine tests were performed using a 32 channel digital EEG machine, the data were collected at least for 30 minutes. Prolonged recordings were performed to acquire data in both sleep and wake state. The child's detail including the presenting complaints, seizure details if present, anti-epilepsy medication, birth related history, early milestone of development, history of any neurological, behavioral or cognitive problems among the family members were meticulously recorded in the laboratory registry book.

The EEG data were reviewed and reported by experienced paediatric neurophysiologists using longitudinal, transverse, average referential, common referential and other montages (SHB). The EEG reports were composed of the child's complaints, neuro-developmental condition, medication and state during the recording, a description part and the comment part. Definite conclusions after electroclinical correlation were reported at the end. The clinicians' question or suspected diagnosis were recorded and addressed in the EEG report.

\section{Demography and clinical criteria}

On confirmation of the diagnosis we reviewed the family residence, referral pattern, the children's age, sex, preexisting medical and neurological conditions, presenting problems, detail of the seizure history including age at first seizure, duration, frequency and age at last attack, initial clinical diagnosis. Detail history related to the child's early psycho-motor development and behavioral state, any major illness or previous chronic disease were also recorded to explore probable underlying cause of the condition.

The primary complaints were recorded and categorized according to the child's functional domains, i.e., regression or deviation from the usual state of the child's motor functional state, posture, speech, behavior, cognitive function and school performance.

History of prior major seizure attack(s) and time of the last attack were categorized as 1) never had any seizure, 2) 48 hours to 4 weeks ago, 3) over 4 to 6 weeks ago, 4) over 6 weeks ago, or, 5) one year ago. Prior seizures type were recorded as complex febrile seizures defined as focal or partial seizure, presence of post attack neurodeficit, lasting for $>15$ minutes or repeated seizures within one febrile episode; nonfebrile generalized tonic clonic seizures; generalized tonic and partial seizures.

Early mile-stone of development was recorded based on the history recalled by the mother ${ }^{9}$ and quick assessment of the child. They were categorized as age appropriate when within normal limit or delayed for the age.

Initial working diagnosis before the EEG were recorded as 'neurodegenerative disorders', 'autistic behaviour / hysteric conversion reaction (HCR)', epilepsy with 'developmental regression', subacute sclerosing panencephalitis (SSPE). None was suspected as NCSE by the referring clinicians.

Subsequently the clinical and investigation findings were correlated to search for the probable cause of NCSE, categorized as 'prolonged seizures' (i.e., Generalized tonic clonic, tonic or clonic seizures lasting for more than 30 minutes); 'poorly controlled epilepsies'; slow viral encephalitis (CMV, herpes simplex virus (HSV)); subacute sclerosing panencephalitis (SSPE) and 'uncertain'.

The EEG feature of continuous or near continuous 1$2.5 \mathrm{c} / \mathrm{s}$ spike wave complexes were categorized into 1) generalized, 2) localized, and 3) localized 
discharges with secondary generalization for further clarification, i.e., to classify the NCSE.

Treatment and outcome:

Specific treatment was started and followed up at one center, Dhaka Shishu Hospital in order to systemic review of the cases. Initially we did not comment on the treatment on first diagnosis, however, advised for a repeat test at about 1 week to 4 weeks later depending on the family condition and the physicians' comfort. We contacted the referring physician over telephone to inform about our interest in reviewing the electro-clinical response to specific treatment protocol of NCSE.

Specific treatment protocol (Chart-1):

After the diagnosis of NCSE, it was the referring physician's decision whether to refer the patient to the study team. Once the child was referred to us, we followed the steps as described bellow:

$1^{\text {st }}$ line of treatment with oral anti-epilepsy drug (AED) Step 1: Children were grouped into a) on single or multiple AED, b) had oral and IV treatment for the present condition, c) not on any AED.

Step 2: All had a repeat EEG before entering to the treatment protocol. Group a: If no significant improvement noted then AEDs were simplified, i.e., oral medication were tapered off into one drug, preferably continue with valproic acid (VPA) or Clobazame. If some response noticed with oral AED, continue with the same drug(s). Group b were advised to follow step 3, Group c were started with oral AED. We used an easily available AED except carbamazepin, as this has been reported to precipitate absence status ${ }^{10}$.

Step 3: Start the $2^{\text {nd }}$ line of treatment with close monitoring on hospital admission. $2^{\text {nd }}$ line of treatment with close monitoring of heart, respiratory rate and blood pressure. We introduced intravenous midazolam (MDZ) (0.15-0.3 mg/kg/hour) for 8 hours on the first day followed by EEG on the next morning, if no change noticed, the infusion was continued for 12 hours. Daily EEG recording for 30 minutes were performed to record the change of continuous discharges.

The courses of infusion continued till 'baseline electroclinical condition', i.e., at least $80 \%$ abolition of the continuous discharges along with return of the baseline clinical condition for the child was achieved. A single oral AED was maintained during the infusion.
In refractory cases, i.e, minor or no improvement after 3 full courses for 12 hours infusion midazolam, oral prednisolone or clobazam was added as adjunctive treatment. Infusion midazolam was tapered off by hours and by dose once significant reduction of continuous spike-wave discharges was noted. The clinical conditions were meticulously reviewed simultaneously.

Step 4: Evaluate the child's electro-clinical state to assess the treatment outcome before discharge from hospital with advise for regular follow up at the epilepsy clinic.

\section{Outcome}

This was recorded as 1) 'good response' when there was return of the baseline electro-clinical condition noted and 2) 'poor response' when there was some improvement but not upto the baseline electroclinical state or no improvement noted on discharge. The outcome of good response were re-categorized for further analysis as 'quick response' (i.e., when target achieved with 1 to 3 courses) and 'delayed response' (when needed 4 and more courses of infusion MDZ).

On discharge from hospital the families were advised to contact at a regular interval and or any time in case of return of the symptoms.

\section{Analysis}

Data were collected in SPSS data base system, descriptive analysis was performed to explore the frequency and criteria of electro-clinical presentation and treatment outcome. ANOVA test was performed to see the correlation between the quick response to treatment and clinical criteria, i.e., prior seizure, motor / speech / behavioural disorder, early milestone of development, EEG criteria and mode of treatment, i.e. oral vs intravenous.

\section{Results}

Forty five children were diagnosed with NCSE in total 3549 EEGs obtained during 5 year 4 month period. Age range was from 5 month to 12 year, mean age 5.4 years, male: female ratio was 1.2: 1 . The majority $(55.5 \%)$ were referred from the in- and out-patient department of Dhaka Shishu (Children's) Hospital (table-I).

Primary complaint was motor functional regression and postural problem in the majority $(37 / 45,82 \%)$ followed by involuntary movement, muscle twitching, jerky movement or unusual frequent fall (31/45, 69\%), speech regression $(29 / 45,64 \%)$ and change of usual behaviour recorded as excessive cry, undue 
demanding attitude, sleepiness, irritability, less responsive, less or excessive active $(25 / 45,60 \%)$, lasting for 1 week to several months (table-II). Motor and postural problems were presented as, slow regression $(17 / 37,46 \%)$ or sudden loss $(18 / 37,49 \%)$ of normal or slow achieved function, $2 / 37,5 \%$ had developmental delay since birth. Eight children had no specific complaint of motor function. Movement disorders were presented as discrete jerks, muscle twitching or head nodding (15/31, 48.4\%), writhing movement (12/ $31,38.9 \%)$ and frequent fall $(4 / 31,18.7 \%)$.

Speech problems presented as total loss of speech $(18 / 29,62 \%)$, regression of articulation, comprehension or irrelevant speech $(11 / 29,38 \%)$, speech developmental delay $(14 / 45,31 \%)$ and no specific complaint reported in $2 / 45,4.4 \%$.

Table-I

Patients profile

\begin{tabular}{lcr}
\hline Item & Number & $\%$ \\
\hline Age & 3 & 5.0 \\
Up to 1 yr & 39 & 92.5 \\
$>1-10 \mathrm{yr}$ & 3 & 2.5 \\
$>10 \mathrm{yr}$ & 45 & 100 \\
$\quad$ Total & & \\
& & \\
Sex & 24 & 52.3 \\
Male & 21 & 47.7 \\
Female & 45 & 100 \\
Total & & \\
& & \\
Residence & 23 & 51.1 \\
Urban & 22 & 48.9 \\
Rural & 45 & 100 \\
Total & & \\
& & \\
Referral pattern & 11 & 24.5 \\
OPD of Dhaka Shishu Hospital \\
CDC of Dhaka Shishu Hospital & 11 & 24.5 \\
IPD of Dhaka Shishu Hospital & 3 & 6.6 \\
Other Hospital & 11 & 24.5 \\
Privet practitioner & 7 & 15.5 \\
Special school & 2 & 4.4 \\
Total & 45 & 100 \\
\hline
\end{tabular}

Table-II

Presenting complaints, clinical condition ( $N=45)$

\begin{tabular}{lrc}
\hline Clinical conditions & Number & $\%$ \\
\hline Primary complaints & & \\
Motor functional regression & 37 & 82.2 \\
Involuntary movement/jerks/fall & 31 & 68.9 \\
Speech regression & 29 & 64.4 \\
Behavioral change & 25 & 55.5 \\
Cognitive functional regression & 23 & 51.1 \\
Regression of school performance & 7 & 15.5
\end{tabular}

History of prior major seizure attacks

$\begin{array}{lrr}\text { Never had } & 13 & 28.8 \\ \text { Seizure } 2 \text { days to 4 weeks ago } & 15 & 33.3 \\ \text { >4 weeks to 6wks ago } & 7 & 15.6 \\ \text { >6 weeks ago } & 7 & 15.6 \\ \text { 1year ago } & 3 & 6.7 \\ \text { Total } & 45 & 100\end{array}$

Prior seizure criteria $(\mathrm{N}-32)$

Complex Febrile Sz* (prolonged/partial) $18 \quad 56.2$

Generalized tonic clonic $\quad 10 \quad 31.2$

$\begin{array}{lll}\text { Generalized tonic } & 5 & 15.6\end{array}$

$\begin{array}{lll}\text { Partial } & 4 & 12.5\end{array}$

Early mile-stone of development

$\begin{array}{lll}\text { Age appropriate } & 21 & 46.6\end{array}$

$\begin{array}{lll}\text { Slow achieved } & 17 & 37.8\end{array}$

$\begin{array}{lll}\text { Moderate delay for the age } & 5 & 11.1\end{array}$

Gross developmental delay $\quad 2 \quad 4.5$

Total $45 \quad 100$

Provisional diagnosis before EEG

$\begin{array}{lll}\text { Neurodegenerative disorder } & 16 & 35.6\end{array}$

Autistic behavior / HCR ${ }^{\star \star} \quad 13 \quad 28.8$

$\begin{array}{lll}\text { Epilepsy with developmental } & 11 & 24.4\end{array}$

regression

$\begin{array}{lll}\mathrm{SSPE}^{\star \star *} & 5 & 11.2\end{array}$

$\begin{array}{lll}\text { Total } & 45 & 100\end{array}$

Probable Cause of NCSE (after some investigation)

\begin{tabular}{lrr} 
Prolonged convulsions & 19 & 42.4 \\
Poorly controlled epilepsy & 10 & 22.2 \\
Slow viral infection, CMV, HSV infection 10 & 22.2 \\
SSPE*** & 4 & 8.8 \\
Uncertain & 2 & 4.4 \\
Total & 45 & 100 \\
\hline
\end{tabular}

* Sz - seizure; ${ }^{*} \mathrm{HCR}$ - hysteric conversion reaction; *** SSPE - subacute sclerosing panencephalitis 
Prior major seizures (generalized tonic clonic, tonic or clonic convulsion) was reported in $32 / 45,71 \%$, within 1 week to 1 year before NCSE was diagnosed (tableII). Among them 22/32, 58.8\% reported the attack 2 days to 6 weeks before the NCSE was diagnosed. The majority $(18 / 32,56.2 \%)$ had complex febrile seizures (table-II). Thirteen children never experienced any major seizure attack, however, had other complaints.

Early mile-stone of developmental skills were age appropriate in $21 / 45,46.6 \%$. None had any contributory history during the pregnancy, history of delayed cry after birth was recorded in 4 children.

Neurodegenerative disorders was recorded as provisional diagnosis in the majority (16/45, 35.6\%), followed by autistic behavior or hysteric conversion reaction (HCR) $(13 / 45,28.8)$.

NCSE were associated with various probable underlying cause, among them the majority (29/45, $64.4 \%$ ) had prolonged convulsions or poorly controlled epilepsy syndromes including Lennox Gastaut Syndrome. One third of the population had evidence of slow viral infection or post infective complication, (i.e., CMV, HSV and measles) (table-II).

CT scan of brain was available in 14 children; 7 revealed abnormality with features of mild atrophy or periventricular leukomalacia and features of old infarction. The EEG feature of generalized continuous slow spike-wave complexes was found in the majority (33/45, 73.3\%) (Table-III, Slide-1).

Treatment and the outcome by steps are represented in chart 1. Six children showed significant electroclinical improvement on oral treatment. They had normal early mile-stone of development, female twice than male, majority were of over 3 years age. Those without noticeable improvement with oral AED $(\mathrm{N}=39)$ were suggested to start with $2^{\text {nd }}$ line of treatment. Out of them 7 children's family declined to start intravenous treatment. At the end total 13 children remained on oral AED and 32 children had protracted IV treatment. Target achieved after 1-3 courses of infusion in the majority (Table-III), 1 family discontinued and lost to follow up. The specific EEG features were presented on diagnosis (Slide-1, 3) and after protracted treatment (Slide-2).
Table-III

Investigation and treatment

\begin{tabular}{lrr}
\hline Item & Number & $\%$ \\
\hline Continuous slow spike wave discharges on EEG: \\
Generalized & 33 & 73.3 \\
Localized & 8 & 17.8 \\
Localized and generalized & 4 & 8.9 \\
Total & 45 & 100
\end{tabular}

Treatment

$1^{\text {st }}$ line treatment before enrolled for this study

$\begin{array}{lrr}\text { Sodium Valproate } & 23 & 57.1 \\ \text { Clobazam } & 16 & 35.5 \\ \text { Phenobarbital } & 8 & 16.0 \\ \text { Nitrazepam } & 3 & 6.6 \\ \text { Clonazepam } & 2 & 4.4 \\ \text { Inj. Phenobarbital or phenytoin } & 4 & 8.8\end{array}$

Treatment

$\begin{array}{lrr}\text { Infusion Midazolam } & 32 & 71.2 \\ \text { Oral AED continued } & 13 & 28.8 \\ \text { Total } & 45 & 100\end{array}$

Number of IV courses required (N-32)

$\begin{array}{lrr}1-3 & 21 & 65.6 \\ 4-6 & 4 & 12.5 \\ 7-10 & 4 & 12.5 \\ >10 & 3 & 9.4\end{array}$

Target assessment

$\begin{array}{lll}\text { Achieved } & 37 & 82.2\end{array}$

$\begin{array}{lll}\text { Not achieved } & 8 & 17.8\end{array}$

Hospital stay (N-45)

\begin{tabular}{lrr}
$1-2$ weeks & 23 & 51.2 \\
$>2$ week-4 weeks & 15 & 33.3 \\
$>30$ day & 2 & 4.4 \\
No hospital stay & 5 & 11.1 \\
\hline
\end{tabular}

Hospital stay was minimum, i.e., upto 2 weeks in 23 children and maximum, i.e., more than 4 wks in 2 children. On multiple logistic backward stepwise regression analysis the dependent variable, i.e, quicker response had shown significant correlation with IV protracted treatment $(p=.000)$, and continuous generalized discharges in the EEG $(p=0.034)$. 
Table-IV

Significant correlation between the quick good response with treatment protocol and EEG feature

\begin{tabular}{|c|c|c|c|c|c|c|c|}
\hline \multirow[t]{2}{*}{ Predictors } & & \multicolumn{3}{|c|}{$\begin{array}{l}\text { Quick response to treatment } \\
\qquad \mathrm{n}=45\end{array}$} & \multicolumn{3}{|c|}{$\begin{array}{c}\text { Multiple Logistic } \\
\text { Regression Analysis }\end{array}$} \\
\hline & & Yes & No & Total & Odds ratio & $\mathrm{Cl}$ & $P$ value \\
\hline \multirow{2}{*}{$\begin{array}{l}\text { Protracted IV } \\
\text { treatment }\end{array}$} & Yes & 20 & 12 & 32 & 4.06 & $0.45-0.82$ & 0.000 \\
\hline & No & 1 & 12 & 13 & & & \\
\hline \multirow{3}{*}{$\begin{array}{l}\text { Generalized } \\
\text { discharges }\end{array}$} & Yes & 14 & 20 & 34 & 1.813 & $1.047-3.140$ & 0.034 \\
\hline & No & 7 & 4 & 11 & & & \\
\hline & Total & 21 & 24 & 45 & & & \\
\hline
\end{tabular}

Chart- I: Flow chart of the conventional and the protracted treatment protocol and outcome

Step 1: Children grouped according to the ongoing AED treatment:

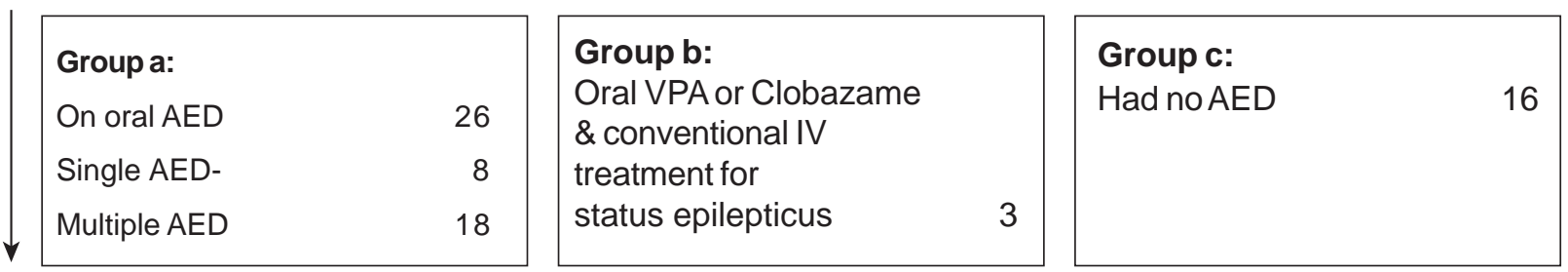

Step 2: Electro-clinical evaluation simplification of ongoing multiple AED therapy or started oral AED

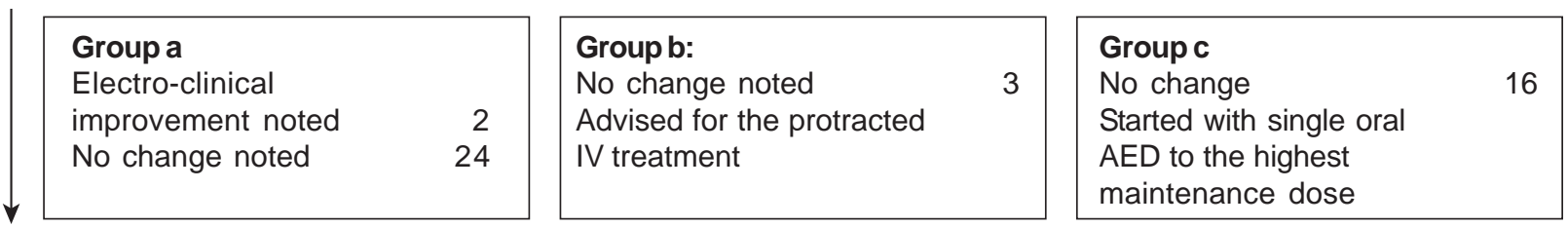

Step 3: Electro-clinical re-evaluation and $2^{\text {nd }}$ line treatment

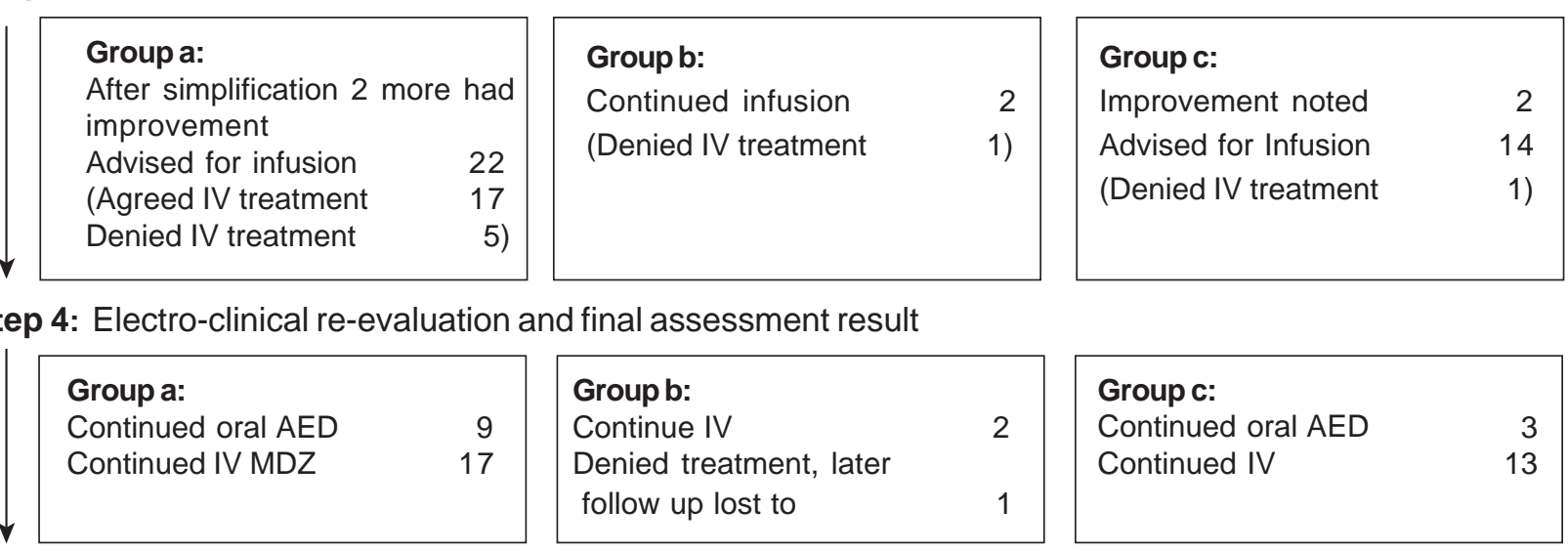

Outcome: Total 39 children were advised for protracted treatment with infusion MDZ 7 did not agree to continue, total 32 continued the infusion

\begin{tabular}{|c|c|}
\hline \multirow[t]{2}{*}{ Oral- } & Good response \\
\hline & Poor response \\
\hline \multirow[t]{3}{*}{ IV- } & Good response \\
\hline & Quick response \\
\hline & Needed $>3$ courses 7 \\
\hline
\end{tabular}

\begin{tabular}{|lr|}
\hline IV & \\
Good response & $2 / 3$ \\
Auick response & 1 \\
Needed $>3$ courses & 1 \\
Lost to FU & 1 \\
\hline
\end{tabular}

\begin{tabular}{|lr|}
\hline Oral AED & \\
Good response & $\mathbf{2 / 3}$ \\
IV: & \\
Good response & $\mathbf{1 2 / 1 3}$ \\
$\quad$ Quick response & 10 \\
$>3$ courses & 2 \\
\hline
\end{tabular}


Nil seen

Slide-1

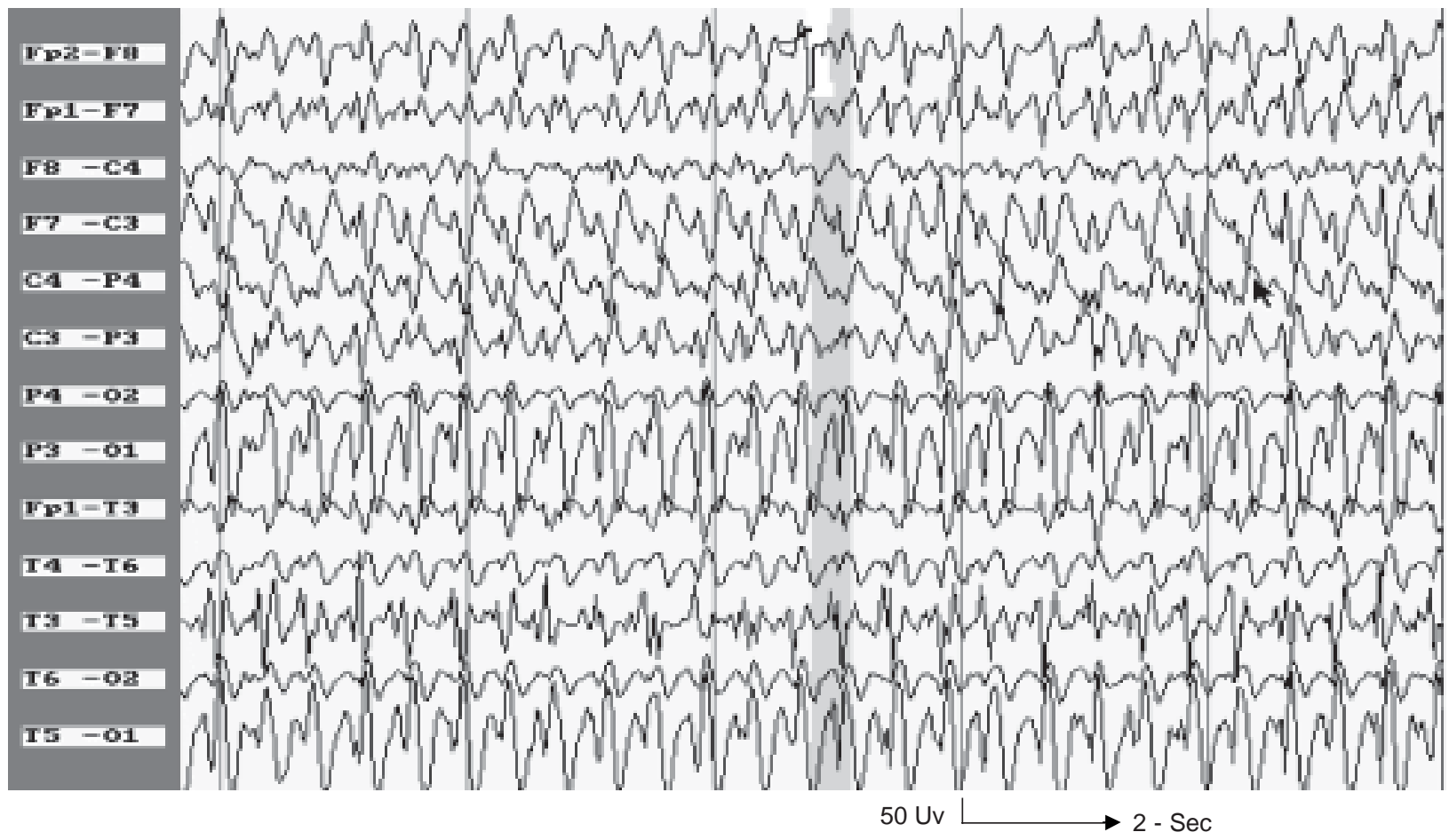

9yr boy, c/o disorientation, unable to sit and walk without help, change of behavior for 10 days, had GTCSz 2 wks ago following a febrile episode, was a school-going child.

\section{Nil seen}

\section{Slide-2}

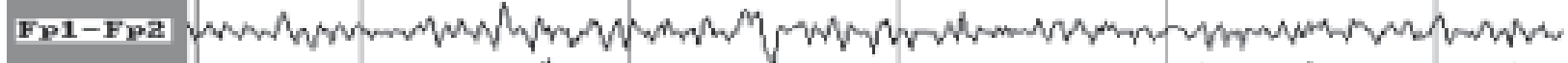
Fp1-F7 Hum

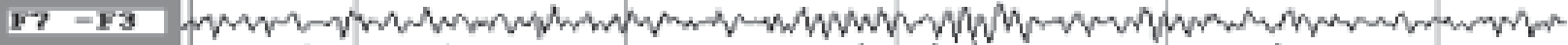

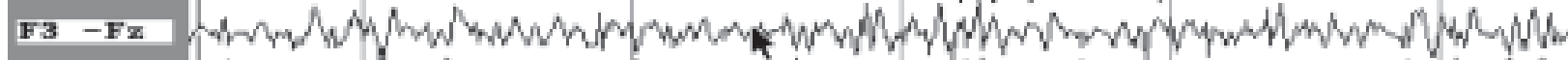

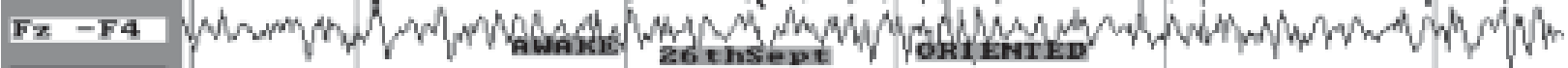
F4 -FB

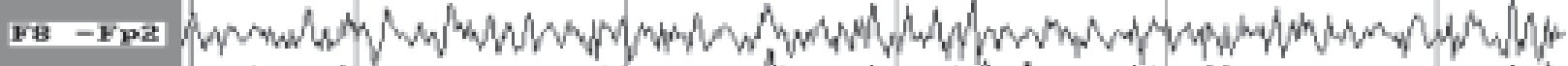

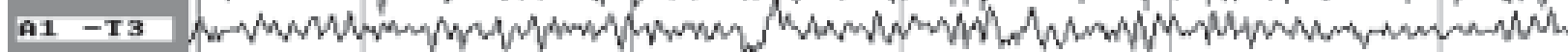

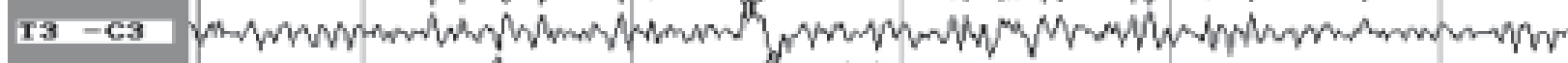

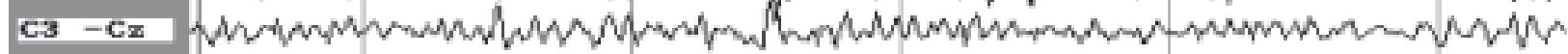

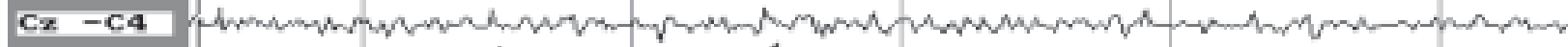

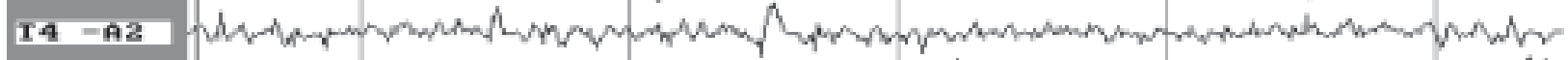

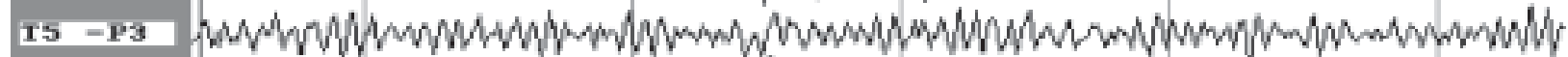

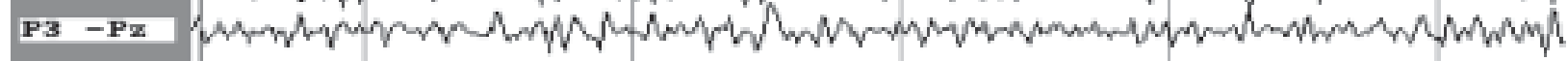

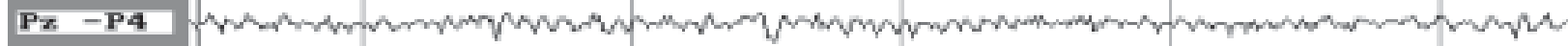

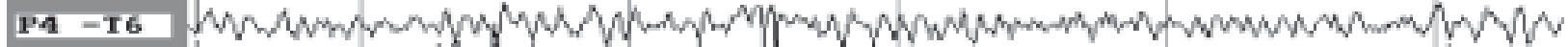

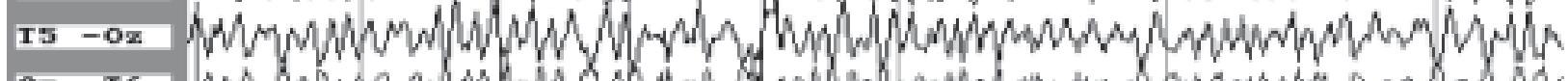

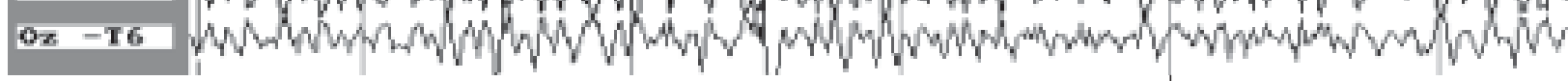
$50 \mathrm{Uv} \longrightarrow 2$ - Sec

Record after $1^{\text {st }}$ dose of infusion MDZ, note normal backgroundactivities and absence of continuous slow spikewave complexes. 
Slide-3

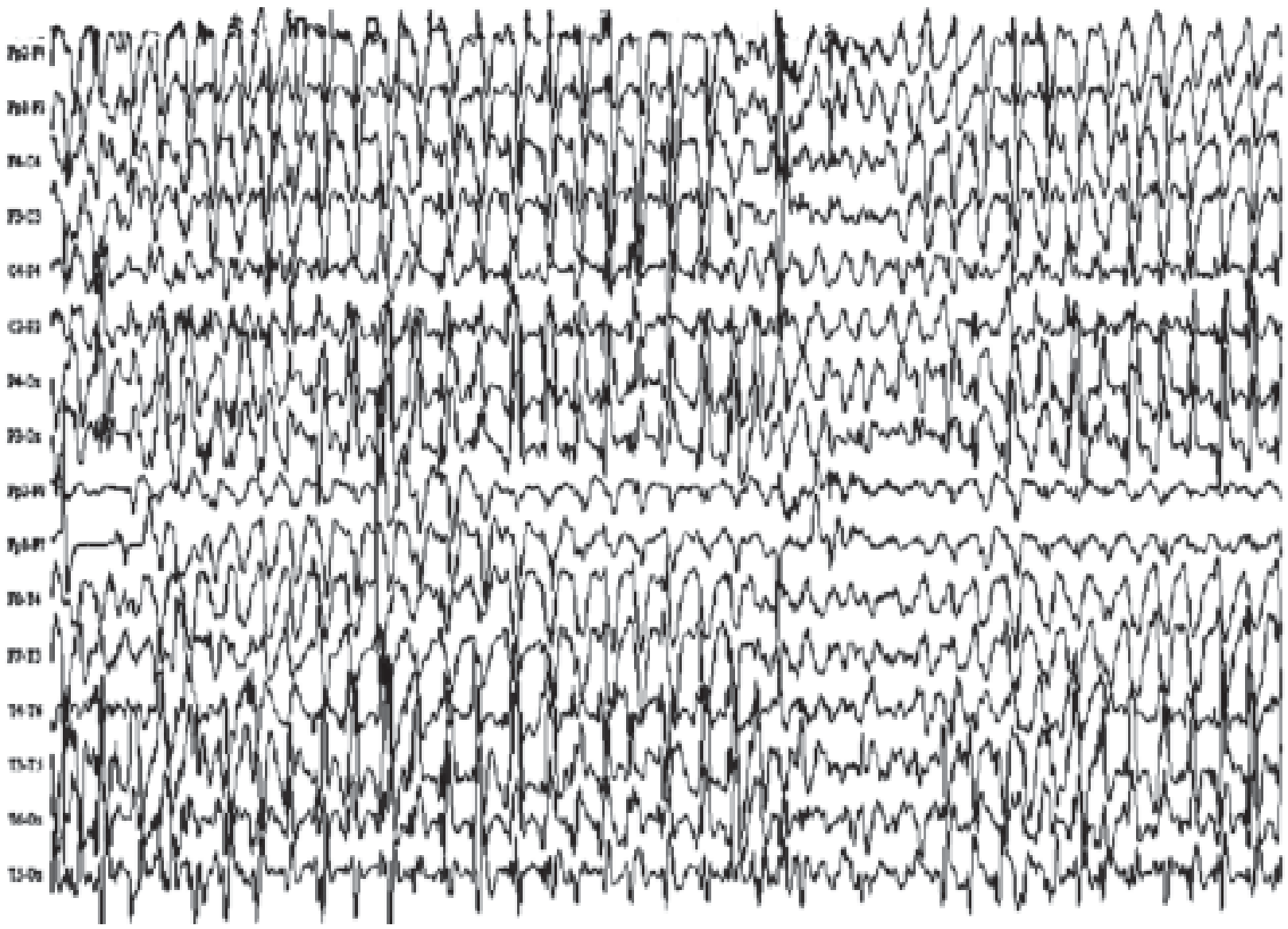

Record of 2year girl with recent loss of motor skills, frequent fall, and jerky movements,speech delay; note continuous $2-2.5 \mathrm{c} / \mathrm{s}$ spike wave complexes with predominance over the fronto-temporal region.

\section{Discussion}

Early recognition of the NCSE condition is a critical goal. The clinical presentation categorized in this study may help the pediatricians to reach that goal. The incidence of NCSE in this region might be higher than what is found in this study. Previous studies suggest that 'routine' EEG will only detect the condition in half the patients with NCSE on continuous EEG monitoring ${ }^{11,12}$. Our population presented with psychomotor, speech and behavioral regression at variable degrees particularly during early childhood to school age. Male female ratio in this study was almost same which was unlike other seizure disorders presented at the children's hospital of the same region ${ }^{13}$. The clinicians definitely had suspected some kind of cerebral dysfunction to be excluded by doing the EEG, however, the primary diagnosis did not direct towards NCSE. Majority had a prior history of seizures within 1 to 4 weeks before the diagnosis of NCSE. About one third had no history of prior major seizure, had unexplained and sustained deterioration of their usual functional state. These finding may bring up an issue of which children should be suspected and monitored for NCSE.

Considering the finding of affected domains in the majority, this may be suggested that children with unexplained regression of motor, speech, cognitive and behavioral state particularly with history of previous seizures should be suspected for NCSE.

Children only with generalized or localized continuous slow-spike wave complexes were included. This is because we wanted to prevent misdiagnosis, identify the treatable disorder at earlier state and to develop a simple guideline. There is higher mortality reported in NCSE with PLED, and GPED ${ }^{6,14-16}$, they should be studied separately.

Oral AEDs and conventional injection of AEDs twice daily was ineffective in the majority. Oral clobazam 
has been suggested by some studies ${ }^{17}$, but was not effective in our case. Continuous infusion of midazolam is found to be effective and safe for NCSE in children while continuous EEG, vital signs and oxygen saturation were monitored during therapy in Japan ${ }^{18}$. In our setting continuous EEG monitoring facility was not available. We therefore tried with the available facility which might be cost-effective.

Midazolam is an imidazobenzodiazepin drug, at physiological $\mathrm{pH}$ the drug becomes lipid soluble and crosses the blood-brain barrier readily. It was introduced in clinical practice in 1982, as a short term sedative for minor operative procedure. The antiepileptic action of midazolam was recognized from the early experimental studies ${ }^{19}$ and effect on EEG was noted by Brown et $\mathrm{al}^{20}$. Published studies suggest minor side-effects such as slight fall in arterial BP and mild bradicardia $^{21}$. There are no published cases of respiratory arrest after intravenous continuous injection in status.

The present protocol with initial oral AED treatment followed by 8 to 12 hours infusion of MDZ and close electro-clinical monitoring probably ensures complete recovery of the status. Return of the baseline electroclinical state needed single course in those with previously normal developmental milestone and apparently shorter duration of NCSE. This finding is supported by other studies ${ }^{22-24}$. On multiple logistic regression analysis quicker response was significantly associated with IV treatment and generalized discharges compared to those with oral treatment and with localized discharges. Early diagnosis and effective treatment with close electro-clinical monitoring is therefore crucial for good outcome.

Failure to recognize the $\mathrm{NCSE}^{25}$ or starting appropriate and prompt treatment is of particular concern with the fear that persistence of the disorder may permanently affect the mental, cognitive and later motor functional stat of the children or might be brain damaging ${ }^{22}$. Appropriate use of EEG in developing countries is essential for diagnosis and prognostic evaluation of seizure disorders is proved in previous studies ${ }^{13,25,26}$.

This study had several limitations including lack of information about the exact duration of the functional regression, and there was no other in-depth investigation available. Neuroimaging was not performed routinely mainly because of financial constrain of the families. Further study with definite protocol is warranted in this region

\section{Conclusion}

This study provides clinical criteria that may guide the clinicians for early suspicion of NCSE in children, which could be confirmed by immediate EEG. A management protocol starting with oral AEDs followed by protracted infusion of MDZ with close electroclinical monitoring and maintenance of long-time AED is found to be effective in this study. Long-term outcome of the same population will be reported in future.

\section{Acknowledgement}

We are grateful to the authority of Comfort Diagnostic centre for their continuous assistance and special care for the children with NCSE, whom they provided service (daily EEG recording) at free of cost. We are also thankful to the authority of Dhaka Shishu Hospital and Child Development and Neurology Unit for their constant support.

\section{References}

1. Kaplan PW. Nonconvulsive status epilepsticus. Neurology 1996; 16: 33-40.

2. Wendy GM. Status epilepticus and acute repetitive seizures in children, adolescents, and young adults: etiology, outcome, and treatment. Epilepsia 1996; 37(suppl): S 74-80.

3. Maganti R, Gerbar P, Corneelia D, Chung S. Nonconvulsive status epilepticus: review. Epilepsy and Behavior 2008; 12: 572-86.

4. Brodtkorv E, Snod T, Kristiansen A, Torbergsen T. Nonconvulsive status epilepticus in the adult mentally retarded. Classification and role of benzodiazepines. Seizure 1993; 2: 115-23.

5. Thomas P, Lebrun C, Chatel M. De Novo absence status epilepticus as a benzodiazepine withdrawal syndrome. Epilepsia 1993; 34: 355-58.

6. Chong DJ, Hirsch LJ. Which EEG patterns warrant treatment in the critically ill? Reviewing the evidence for treatment of periodic epileptiform discharges and related patterns. Journal of Clinical Neurophysiology 2005; 22: 79-91.

7. Snodgrass SM. Clinical significance of periodic lateralized epileptiform discharges: relationship with status epilepticus. Journal of Clinical Neurophysiology 1989; 16: 159-72. 
8. Tay SK, Hirsch JL, Leary L, Jette N, Wsittman J, Akmen IC. Nonconvulsive status epilepticus in children: clinical and EEG characteristics. Epilepsia 2006; 47: 1504-09.

9. Zaman SZ, Khan NZ, Islam S, Banu S, Dixit S, Shrout $\mathrm{P}$, et al. Validity of the 'Ten question' for screening serious childhood disability: result from urban Bangladesh. Int J Epidemiol 1990; 19: 613-20.

10. Callahan DJ, Noetzel MJ. Prolonged absence status epilepticus associated with carbamazepine therapy, increased intracranial pressure, and transient MRI abnormalities. Neurology 1992; 42: 2198-2201.

11. Claassen JM, Mayer SA, Kowalski RG. Detection of electrographic seizures with continuous EEG monitoring in critically ill patients. Neurology 2004; 62: 1743-48.

12. Pandian JD, Cascino GD, So EL. Digital video electroencephalographic monitoring in the neirological-neurosurgical intensive care unit: Clinical feature and outcome. Archive of Neurology 2004; 61: 1090-94.

13. Banu SH, Khan NZ, Hossain M, JahanA, Parveen M, Rahman N, et al. Profile of childhood epilepsy in Bangladesh. Developmental Medicine and Child Neurology 2003; 45: 477-82.

14. Engel JJ, Ladwig B, Fetell M. Prolonged partial somplex status epilepticus. EEG and behavioral observations. Neurology 1978; 28: 863-69.

15. Krsek P, Mikulecka A, Druga R. Longterm behavioral and morphological consequences of non-convulsive status epilepticus in rats. Epilepsy Behavior 2004; 5: 180-91.

16. Stancey KHT, Lawrence JH, Linda L, Nathalie J, John W, Gigdem IA. Non-convulsive status epilepticus in children: Clinical and EEG characteristics. Epilepsia 2006; 47: 1504-09.
17. Walker MC. Diagnosis and treatment of nonconvulsive status epilepticus. CNS Drugs 2001; 15: 931-39.

18. Tutsuro N, Kenji S, Michio F, Shigeru F, Masayuki S. Treatment of NCSE in children. No to Hattatsu 2005; 37: 369-73.

19. de Jong RH, Bonin JD, Benzodiazepines protect mice from local anesthetic convulsions and death. Anesthesia and Analgesia 2009; 60: 38589.

20. Brown CR, Sarnquist FH, Canup CA, Pedley TA. Clinical electroencephalographic and pharmacokinetic studies of a water-soluble benzodiazepine, midazolam maleate. Anesthesiology 1979; 50: 467-70.

21. Ghilain S, Van RKJ, De Barsy TH. Midazomal in the treatment of epileptic seizures. Neurology, Neurosurgery and Psychiatry 1988; 51: 732.

22. Krumholz A, Sung GY, Fisher RS, Barry E, Bergey GK, Grattan LM. Complex partial status epilepticus accompanied by serious morbidity and mortality. Neurology 1995; 45: 1499-1504.

23. Murthy JMK. Nonconvulsive Status Epilepticus. An underdiagnosed and potentially treatable condition. Neurol India 2005; 51: 453-54.

24. Stores G, Zaiwella Z, Styles E, Hoshika A. Nonconvulsive status epilepticus. Arch Dis Child 1995; 73: 106-11.

25. Banu SH, Hussain M, Khan NZ. Non-convulsive status epilepticus presenting as deteriorating schood performance - case report. Bangladesh Journal of Child Health 1998; 22: 75-77.

26. Banu SH, Hossain M, Khan N. Two year experience of developing Paediatric EEG services in Bangladesh: Report of the first 1000 cases [abstract]. Journal of Electrophysiological Technology 1999; 25: 56. 